The use of video capsule ileoscopy in pediatric patients is becoming more common. The technique has been used in cases of obscure small-bowel bleeding, polyposis, Crohn's disease, and, occasionally, to investigate the anatomical anomalies of the gastrointestinal tract that are typically encountered in this age group $[1,2]$.

We report here the case of a 15-year-old patient with symptoms characteristic of intestinal bleeding and severe anemia, who required repeated transfusions. The hematochemical parameters (erythrocyte sedimentation rate, CRP (c-reactive protein), antineutrophil cytoplasmic antibodies, anti-Saccharomyces cerevisiae antibodies (ASCA), antiendomysium antibodies, and the transglutaminases), and esophagogastroduodenoscopy and colonoscopy examinations, including histology, were normal.

Suspecting a Meckel's diverticulum, a scintigraphic evaluation was performed, which showed an accumulation of tracer in the gastric area, anomalously located at the level of the hypochondrium on the right side. Video capsule endoscopy revealed a small umbilicated mass projecting into the lumen at the level of the mid-ileum (Figure 1). At surgery, the young patient was found to have intestinal duplication associated with intestinal malrotation (Figure $\mathbf{2}$ ), and the diagnosis was subsequently confirmed histologically (Figure 3).

Ileoscopy using a video capsule is a wellknown technique for the investigation of adult patients, but it can also be used in the pediatric age group. It is a very useful tool for the diagnosis of jejuno-ileal disease (e.g. polyposis, Crohn's disease, Meckel's diverticulum) due to its high sensitivity and specificity in comparison with traditional radiological techniques. A uniform iconography should be adopted for a better endoscopic definition of these diseases, which, though rare, can be clear-

\title{
Video capsule diagnosis of intestinal duplication in a 15-year-old patient
}

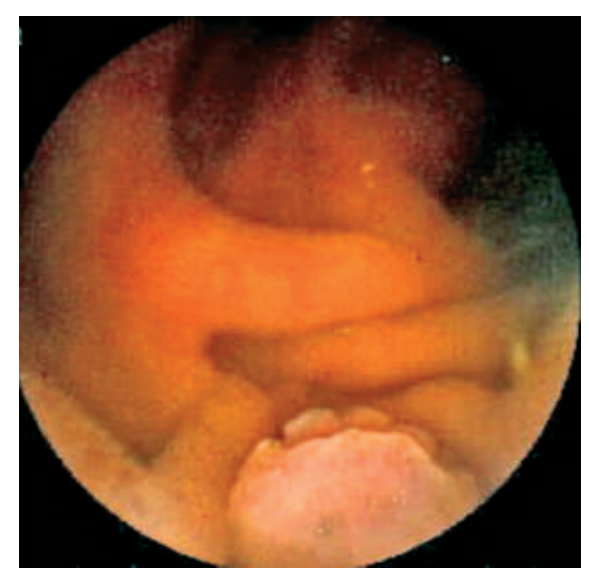

Figure 1 A video capsule image showing an umbilicated mass projecting into the lumen at the level of the mid-ileum.

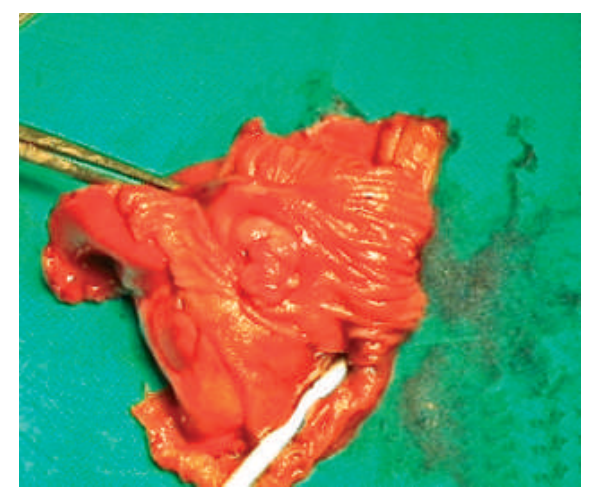

Figure 2 The surgical specimen, showing an intestinal duplication. The communication between the intestinal lumen and the duplication is clearly visible.

ly detected using the video capsule technique [3].

Endoscopy_UCTN_Code_CCL_1AC_2AF
F. Torroni', P. De Angelis', T. Caldaro', G. Federici', A. Pane', C. Romano², L. Dall'Oglio ${ }^{1}$
${ }^{1}$ Digestive Surgery and Endoscopy Unit, Ospedale Pediatrico Bambino Gesù, Rome, Italy
2 Policlinico Universitario, University of Messina, Messina, Italy.

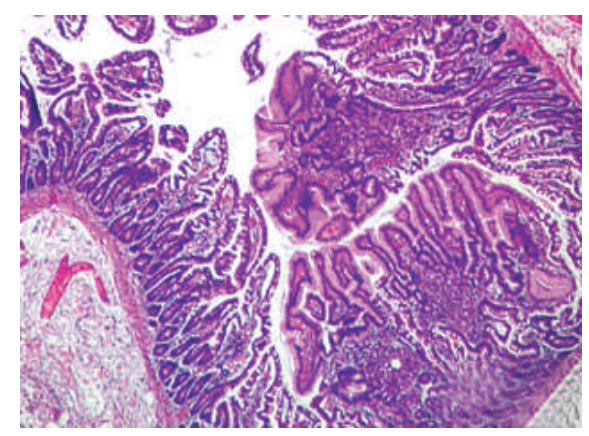

Figure 3 Histological view showing intestinal mucosa, with typical villi, on the lefthand side, and gastric mucosa on the right.

\section{References}

${ }^{1}$ Swain P, Fritscher-Ravens A. Role of video endoscopy in managing small-bowel disease. Gut 2004; 53: 1866- 1875

2 Viazis N, Papaxoinis K, Theodoropoulos Iet al. Impact of capsule endoscopy in obscure small-bowel bleeding: defining strict diagnostic criteria for a favorable outcome. Gastrointest Endosc 2005; 62: 717 - 722

${ }_{3}$ Pennazio M, Santucci R, Rondonotti Eet al. Outcome of patients with obscure gastrointestinal bleeding after capsule endoscopy: report of 100 consecutive cases. Gastroenterology 2004; 126: 643-653

\section{Corresponding author}

\section{F. Torroni, M. D.}

Ospedale Pediatrico Bambino Gesù Piazza S. Onofrio 4

\section{Roma}

Italy

Fax: $\quad+39-0668592543$

E-mail: ftorroni@yahoo.it 